\title{
INFLUENCIA DE LOS PARÁMETROS DE DEPOSICIÓN EN LA COMPOSICIÓN DE RECUBRIMIENTOS DE ZnCo
}

\author{
INFLUENCE OF THE DEPOSITION PARAMETERS ON THE ZnCo COATINGS \\ COMPOSITION
}

\section{Célia Regina Tomachuk Dos Santos Catuogno}

Lic. en Cs. Quimicas, Dr. en Ing. Mecánica, Investigadora, Instituto de Pesquisas Energéticas y Nucleares, IPEN/CNEN-SP, CCTM, Av. Prof. Lineu Prestes, 2242, CEP 05508-000, São Paulo, SP, Brazil, tomazuk@gmail.com

Alejandro Ramón Di Sarli

Ing. Qco., Dr. en Ing., Investigador y Director, Centro de Investigación y Desarrollo en Tecnología de Pinturas (CICPBA-CONICET); Av. 52 s/n entre 121 y 122. CP. B1900AYB, La Plata - Argentina dirección@cidepint.gov.ar

Fecha de recepción: 23 abril de 2010

Fecha de aprobación: 23 mayo de 2010

\section{RESUMEN}

En el presente trabajo se estudió la morfología y la estructura cristalina de electrodepósitos de $\mathrm{ZnCo}$, obtenidos mediante la aplicación de corriente modulada o corriente continua. El baño de deposición utilizado contenía iones cloruro y eran de carácter ácido. La morfología y la estructura cristalina del depósito fueron analizadas mediante Microscopia Electrónica de Barrido y Difracción de Rayos X, respectivamente, mientras que su composición química se obtuvo, utilizando Inductively Coupled Plasma Spectroscopy (ICP). Los resultados experimentales mostraron que la morfología de los codepósitos era independiente de los parámetros de deposición, más no su contenido de cobalto. Los codepósitos obtenidos con corriente modulada, presentaron mayor concentración de cobalto y orientación cristalina diferente de aquéllos generados con corriente continua. La cantidad de aditivos necesaria en el baño, fue menor cuando se utilizó corriente modulada.

Palabras clave: recubrimientos $\mathrm{ZnCo}$, electrodeposición, corriente modulada 


\section{ABSTRACT}

The main purpose of this paper was to study the morphology and crystalline structure of a $\mathrm{ZnCo}$ coating achieved by pulsed current electroplating and comparing them with those deposits prepared using direct current. The plating bath contained chloride ions and acid $\mathrm{pH}$. The deposit crystalline morphology and structure was investigated by means of Scanning Electron Microscopy and X Ray Diffraction respectively, but the zinc quantity was determined by Inductively Coupled Plasma Spectroscopy. The experimental results showed free-deposition parameters for deposit morphology but not its cobalt content. Pulsed current deposits showed higher content of $\mathrm{Co}$ and crystalline orientation different of that obtained using direct current. The additive quantity required in the bath was smaller using pulsed current.

Key words: ZnCo coatings, electrodeposition, pulsed current.

\section{INTRODUCCIÓN}

En los últimos 20 años, la deposición electrolítica del zinc tuvo un gran desarrollo debido a la demanda de superficies metálicas más resistentes a la corrosión por parte de la industria automotriz [1]. Entre las innovaciones, una de las más importantes fue la deposición de aleaciones de zinc, tales como $\mathrm{ZnFe}, \mathrm{ZnCo}$ y $\mathrm{ZnNi}$, las cuales dieron origen a un recubrimiento electroquímicamente más noble que el de zinc puro [2] y, por lo tanto, con menor velocidad de corrosión.

Si el contenido de un elemento más noble resulta muy bajo, como por ejemplo en el caso de aleaciones $\mathrm{ZnCo}$ o $\mathrm{ZnFe}$ con contenido de Fe y Co menor que 1\%, la mayor resistencia a la corrosión de la aleación, comparada con la del zinc puro, es atribuida a su estructura cristalina, la cual presenta un crecimiento preferencial de planos reticulares [3].

Con respecto de la resistencia a la corrosión, algunos autores [4,5] creen que la aleación ZnCo es la más prometedora para sustituir el zinc en la industria automotriz, pues con concentraciones de Co entre $0,6 \%$ y $1,0 \%$ se obtienen recubrimientos superiores a los de zinc puro y comparables a los de $\mathrm{ZnNi}$ (15\% de Ni). Estos recubrimientos de $\mathrm{ZnCo}$ son producidos a partir de baños ácidos a base de cloruro [5] o alcalinos libres de cianuro[6]; un posterior tratamiento de pasivación, mejora sus propiedades de resistencia a la corrosión.

Desde el punto de vista práctico y económico, la deposición de aleaciones presenta mayores dificultades de manejo y es más cara que la de zinc puro. Sin embargo, también 
es verdad que el mismo grado de protección que ofrece un recubrimiento de zinc puro, es alcanzado con un menor espesor de la aleación [7].

El uso de corriente pulsante es cada vez más extendido como técnica de electrodeposición de metales y aleaciones [8], porque sus depósitos poseen granulometría más fina, son más compactos [9,11], libres de porosidad, y presentan bajo nivel de inclusiones [12]. Vale resaltar que la técnica de corriente pulsante, ha sido establecida debido a su mayor eficacia para controlar la estructura y las propiedades de los electrodepósitos.

En la electrodeposición convencional con corriente continua, sólo un parámetro, la densidad de corriente, puede ser modificado. En cambio, al utilizar métodos pulsantes, es posible controlar cuatro parámetros importantes: (a) la densidad de corriente de pulso catódico $\left(\mathrm{j}_{\mathrm{p}}\right)$; (b) la densidad de corriente de pulso anódico o nulo; (c) la duración del pulso catódico $\left(\mathrm{t}_{\mathrm{on}}=\right.$ on-time); $\mathrm{y}(\mathrm{d})$ la duración del pulso anódico o nulo ( $\mathrm{t}_{\text {off }}=$ offtime) [9]. La suma de los on-time y off-time, constituye un ciclo (duty cicle), conforme con lo que muestra en la figura 1. El duty cicle puede variar de $1 \%$ a $100 \%$, siendo que el $100 \%$ corresponde a corriente continua convencional, debido a que no existe off-time.

Duty cycle $=($ on-time/on-time + off-time $) \times 100$

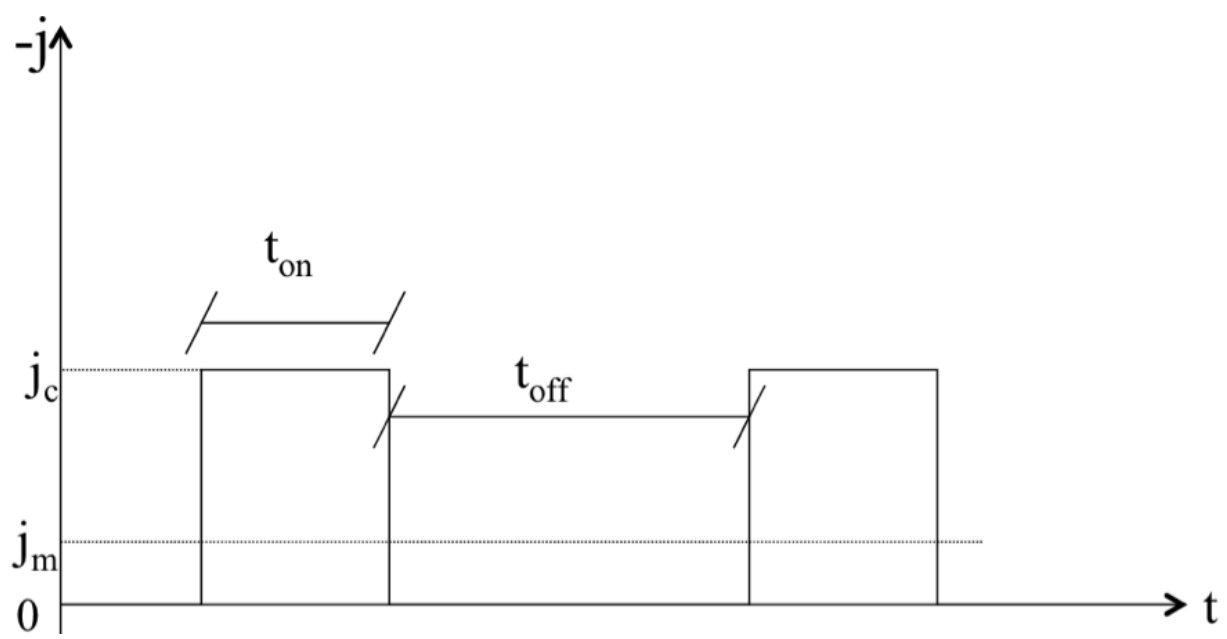

Figura 1. Parámetros de la corriente pulsante

La densidad de corriente media $\left(\mathrm{j}_{\mathrm{m}}\right)$ es equivalente a la densidad de corriente aplicada en la electrodeposición con corriente continua [13], y se la define como:

$$
\mathrm{j}_{\mathrm{m}}=\mathrm{j}_{\mathrm{p}} \times \mathrm{t}_{\mathrm{on}} /\left(\mathrm{t}_{\mathrm{on}}+\mathrm{t}_{\text {off }}\right)
$$


Donde $\mathrm{t}_{\text {on }} \mathrm{yt}_{\text {off }}$ son, respectivamente, la duración de los pulsos catódico y anódico, figura 1. Sin embargo, debido a que cada sistema ofrece una respuesta diferente, no sólo es difícil prever la influencia de cada uno de esos parámetros, sino también encontrar las condiciones ideales de electrodeposición para obtener un recubrimiento con las propiedades deseadas. Los objetivos del presente trabajo fueron:

a) investigar las modificaciones estructurales y morfológicas de electrodepósitos de $\mathrm{ZnCo}$ (con concentraciones de Co entre $0,4 \%$ y $1,2 \%$ ), preparados mediante el uso de parámetros de pulso bien definidos.

b) emplear la técnica de electrodeposición con corriente pulsante con el fin de obtener electrodepósitos de $\mathrm{ZnCo}$ de grano fino, utilizando una concentración de aditivos menor al $50 \%$ de la recomendada por el fabricante del baño. Los resultados serán comparados con electrodepósitos de $\mathrm{ZnCo}$ conseguidos con una densidad de corriente equivalente a la densidad de corriente media de la técnica de electrodeposición con corriente pulsante, y con $100 \%$ de los aditivos recomendados en el boletín técnico entregado por el proveedor de los productos.

\section{PROCEDIMIENTO EXPERIMENTAL}

Para los ensayos de electrodeposición fue utilizada una celda electroquímica convencional con tres electrodos y capacidad de $1 \mathrm{dm}^{3}$. El electrodo de referencia, fabricado especialmente con esa finalidad, era de cobalto puro y su potencial, con respecto de un electrodo de referencia de $\mathrm{Ag} / \mathrm{AgCl}$, fue medido antes de cada experimento; el contra-electrodo era una lámina de zinc puro recubierta con una capa de cobalto para evitar que se consumiera el cobalto del baño; finalmente, como electrodos de trabajo, se usaron discos de acero con bajo tenor de carbono, desbastados hasta malla 1.200, secados con aire comprimido, colocados en un porta-muestra de acrílico, dejando un área expuesta de $4 \mathrm{~cm}^{2}$. Antes de la electro-deposición, las muestras fueron pesadas, tratadas con electrólisis anódica y catódica durante dos minutos en solución de $\mathrm{NaOH} 60$ g. $\mathrm{L}^{-1}$ a $4 \mathrm{~V}$, neutralizadas en solución $2 \%$ de $\mathrm{HCl}$, lavadas con agua destilada, secadas con aire y nuevamente pesadas.

El electrolito utilizado fue: $3,5 \mathrm{~g} \cdot \mathrm{L}^{-1} \mathrm{CoCl}_{2} \cdot 6 \mathrm{H}_{2} \mathrm{O}, 77,2 \mathrm{~g} \cdot \mathrm{L}^{-1} \mathrm{ZnCl}_{2}, 220 \mathrm{~g} \cdot \mathrm{L}^{-1} \mathrm{KCl}, 26 \mathrm{~g} \cdot \mathrm{L}^{-1}$ $\mathrm{H}_{3} \mathrm{BO}_{3}$ y agentes aditivos ( $15 \mathrm{~mL}$ de surfactante y $2 \mathrm{~mL}$ de abrillantador provistos por la empresa UNISIDA-Europlating de Milán, Italia), la temperatura de $30^{\circ} \mathrm{C}$ y el pH 5,5 . Todas las soluciones fueron preparadas con reactivos puros pro-análisis y agua destilada. Durante la electro-deposición, la solución fue burbujeada con aire.

En la electro-deposición con corriente continua, la densidad de corriente (j) fue variada de 5 a $40 \mathrm{~mA} . \mathrm{cm}^{-2}$ mientras que con corriente modulada, los parámetros tuvieron la 
siguiente variación: densidad de corriente de pulso $\left(j_{p}\right)=250 \mathrm{~mA} \cdot \mathrm{cm}^{-2}$; densidad de corriente media $\left(\mathrm{j}_{\mathrm{m}}\right)$ de 5 a $40 \mathrm{~mA} . \mathrm{cm}^{-2}$; off-time $\left(\mathrm{t}_{\text {off }}\right)$ de 5 a $16,8 \mathrm{~ms}$; on-time $\left(\mathrm{t}_{\mathrm{on}}\right)$ de 1 a 3,2 ms. El duty cycle que es la relación entre el on-time y el off-time, permaneció fijo en $16 \%$. El duty cycle fue definido de acuerdo con lo realizado por W. Paasth [14] en su trabajo relacionado con la electrodeposición de aleaciones $\mathrm{ZnCo}$, mediante corriente pulsante. El tiempo de deposición fue calculado para obtener un espesor promedio de $25 \mu \mathrm{m}$.

Los depósitos con corriente continua fueron realizados, usando un potenciostato 549 AMEL y con corriente modulada, un potenciostato 2055 AMEL acoplado a un generador de función programable 568 AMEL. La forma de la señal aplicada fue observada con un osciloscopio digital de Nicolet Instrument Corporation modelo 210, y los transientes de corriente, impresos en un registrador X-Y, Olivetti modelo 600.

El contenido de Zn y Co en los depósitos, fue determinado, disolviendo estos últimos en una solución 1:3 de $\mathrm{HCl}$, que luego fue analizada mediante un Espectrómetro de Emisión de Acoplamiento Inductivo (ICP por sus siglas en inglés), de Perkin Elmer modelo 5005. La morfología de las muestras se observó mediante Microscopía Electrónica de Barrido con sonda para microanálisis (SEM/EDXS), Philips modelo XL 20, mientras que las fases presentes fueron caracterizadas con Difracción de Rayos X (XRD), en un equipo Philips modelo PW 1730, utilizando radiación incidente CuK $\alpha(\lambda=15,4 \mathrm{~nm}$, $40 \mathrm{KV}$ y $30 \mathrm{~mA}$ ), y barrido angular de $1 \% / \mathrm{min}$ a partir de $20^{\circ}$ y hasta $85^{\circ}$ en $2 \theta$. Las correspondientes posiciones angulares y distancias interplanares fueron calculadas a partir de la Ley de Bragg y los picos identificados con las micrografías provistas por el Powder Diffraction File (PDF), organizado por el Joint Committe on Powder Diffraction Standards (JCPDS).

Las medidas del espesor de la capa de revestimiento obtenida, fueron realizadas con un medidor de espesor digital Helmut Fischer modelo Deltascope MP2.

Los ensayos de microdureza Vickers basados en la norma ASTM E384-10e2, fueron ejecutados en recubrimientos con espesor mayor que $25 \mu \mathrm{m}$ y exentos de microporos mediante un microdurómetro Leitz-Wetzlar. Las impresiones fueron obtenidas con cargas de $25 \mathrm{~g}$ en la sección transversal del recubrimiento. La preparación de las muestras consistió en fracturar con nitrógeno líquido para evitar posibles contaminaciones del sustrato, embutido en bakelita, lijado hasta grano 1200, limpieza con acetona en ultrasonido, secado, pulido con pasta de diamante hasta $1 \mu \mathrm{m}$, limpieza con acetona en ultrasonido y secado.

\section{RESULTADOS Y DISCUSIÓN}

Recubrimientos de ZnCo (contenido de Co entre 0,4\% y 1,2\%), fueron obtenidos, utilizando un baño con $3 \%$ de iones cobalto. El electrolito usado en la electrodeposición 
con corriente pulsante, contenía una concentración de aditivos $50 \%$ menor que la recomendada por el fabricante del baño (este es uno de los objetivos propuestos en el trabajo), en tanto que la concentración de aditivo en el electrolito empleado para la electrodeposición con corriente continua, era del $100 \%$.

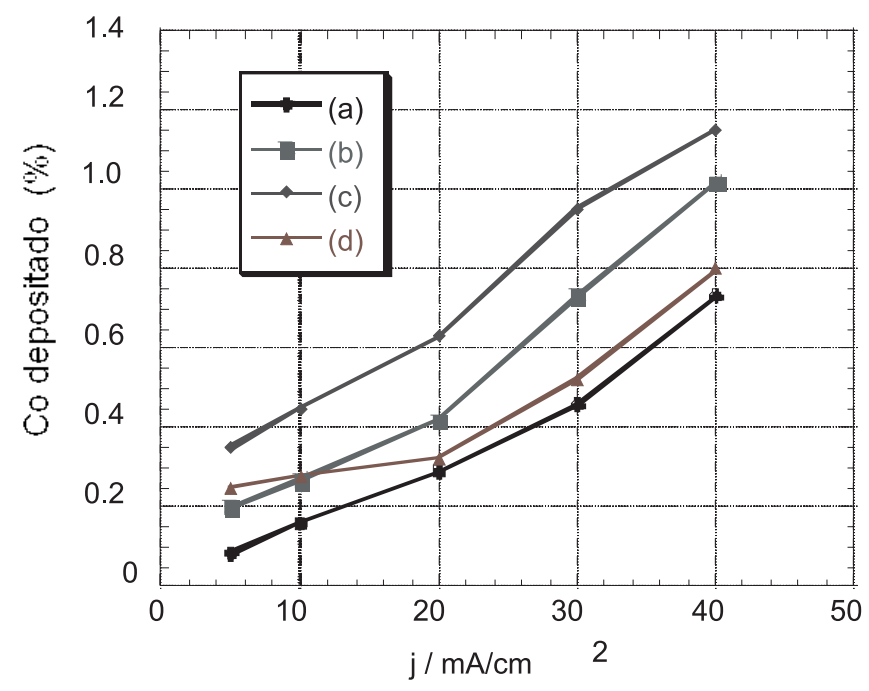

Figura 2. Efecto de la densidad de corriente en relación sobre el contenido de Co en la aleación (a) $\mathrm{t}_{\text {on }}=1 \mathrm{~ms} \mathrm{y}_{\text {off }}=5 \mathrm{~ms}$; (b) $\mathrm{t}_{\text {on }}=1,5 \mathrm{~ms} \mathrm{y}_{\text {off }}=8,5 \mathrm{~ms}$; (c) $\mathrm{t}_{\text {on }}=3,2 \mathrm{~ms} \mathrm{y}_{\text {off }}=16,8 \mathrm{~ms}$; (d) corriente

La figura 2 presenta las diferentes condiciones de electrodeposición utilizadas en la electrodeposición con corriente pulsante y descritas en la tabla 1. El ciclo de trabajo permaneció en $16 \%$. El electrolito contenía $3 \%$ de cobalto y aditivos, $\mathrm{pH}=5,5$ y la temperatura era de $30^{\circ} \mathrm{C}$.

Tabla 1. Parámetros de electrodeposición en función de la composición y microdureza de los electrodepósitos de ZnCo.

\begin{tabular}{|c|c|c|c|c|}
\hline Identificación & $\begin{array}{c}\text { On-time } \\
(\mathbf{m s})\end{array}$ & $\begin{array}{c}\text { Off-time } \\
(\mathbf{m s})\end{array}$ & $\begin{array}{c}\mathbf{C o}_{\mathrm{d}} \\
\mathbf{( \% )}\end{array}$ & $\begin{array}{c}\text { Microdureza } \\
(\mathbf{H V})\end{array}$ \\
\hline $\mathrm{a}$ & 1 & 5 & 0,45 & 88,5 \\
\hline $\mathrm{b}$ & 1,5 & 8,5 & 0,75 & 116 \\
\hline $\mathrm{c}$ & 3,2 & 16,8 & 0,90 & 123 \\
\hline $\mathrm{d}$ & \multicolumn{2}{|r|}{ Corriente continua } & 0,50 & 90 \\
\hline
\end{tabular}

Se verificó (figura 2), que en el mismo electrolito era posible obtener recubrimientos de $\mathrm{ZnCo} \mathrm{con} \mathrm{mayor} \mathrm{porcentaje} \mathrm{de} \mathrm{cobalto,} \mathrm{con} \mathrm{sólo} \mathrm{variar} \mathrm{apenas} \mathrm{los} \mathrm{parámetros} \mathrm{del}$ proceso de electro-deposición. La condición (c), $\mathrm{t}_{\mathrm{on}}=3,2 \mathrm{~ms}$ y $\mathrm{t}_{\text {off }}=16,8 \mathrm{~ms}$ es la que presentó mejores resultados, pues contenidos de $\mathrm{Co}<0,4 \%$ no mejoran las propiedades protectoras de los recubrimientos [17]. 
Las imágenes de SEM, figuras 3a, 3b y 3c, muestran la superficie del co-depósito de $\mathrm{ZnCo}$ obtenida con corriente pulsante, densidad de corriente media de $30 \mathrm{~mA} . \mathrm{cm}^{-2}$, corriente modulada y los siguientes parámetros de deposición: $\mathrm{t}_{\text {on }}=1 \mathrm{~ms}$ y $\mathrm{t}_{\text {off }}=5 \mathrm{~ms}$;

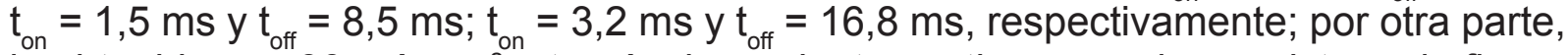
la obtenida con $30 \mathrm{~mA} \cdot \mathrm{cm}^{-2}$ a través de corriente continua, puede ser vista en la figura $3 d$. En ellas, puede verse que: 1 ) los recubrimientos obtenidos con corriente modulada presentaban granulometría más fina que los generados con corriente continua; 2 ) los codepósitos no presentan micro-porosidades; y 3) la apariencia de todos los recubrimientos obtenidos fue cenicienta, brillante y con adherencia satisfactoria, conforme con la norma ASTM B571-97(2008)e1.

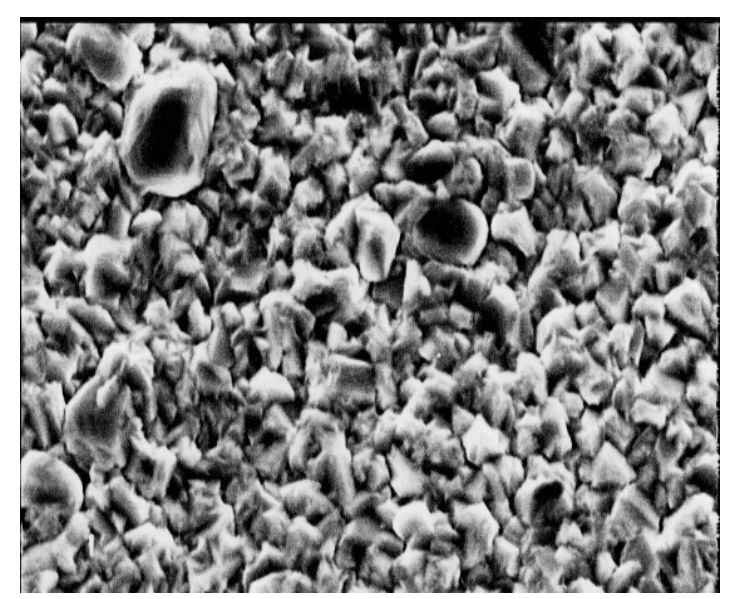

(a) corriente modulada, $\mathrm{Co}_{\mathrm{d}}=0,45 \%$

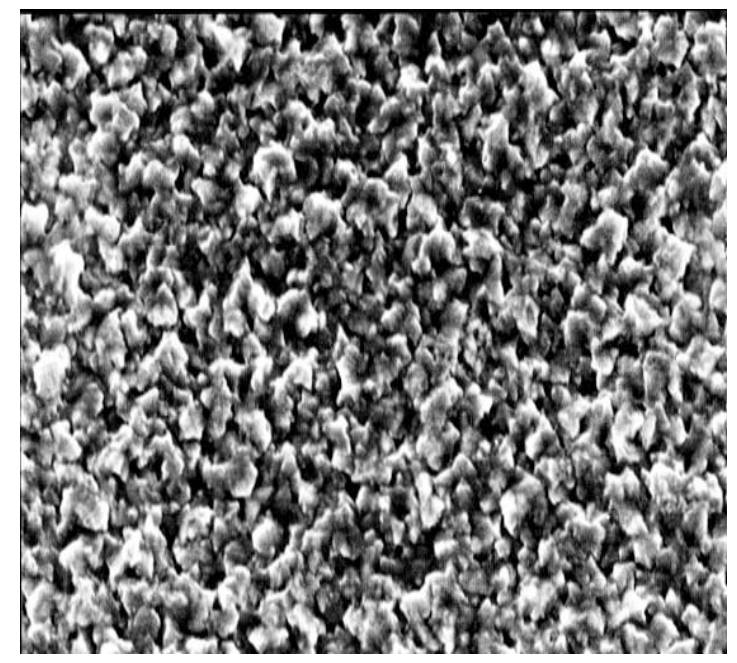

(c) corriente modulada, $\mathrm{Co}_{\mathrm{d}}=0,90 \%$

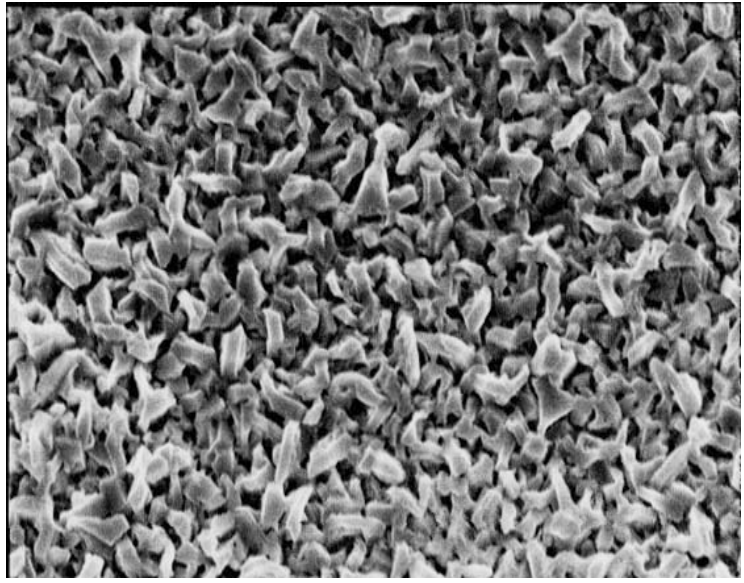

(b) corriente modulada, $\mathrm{Co}_{\mathrm{d}}=0,75 \%$

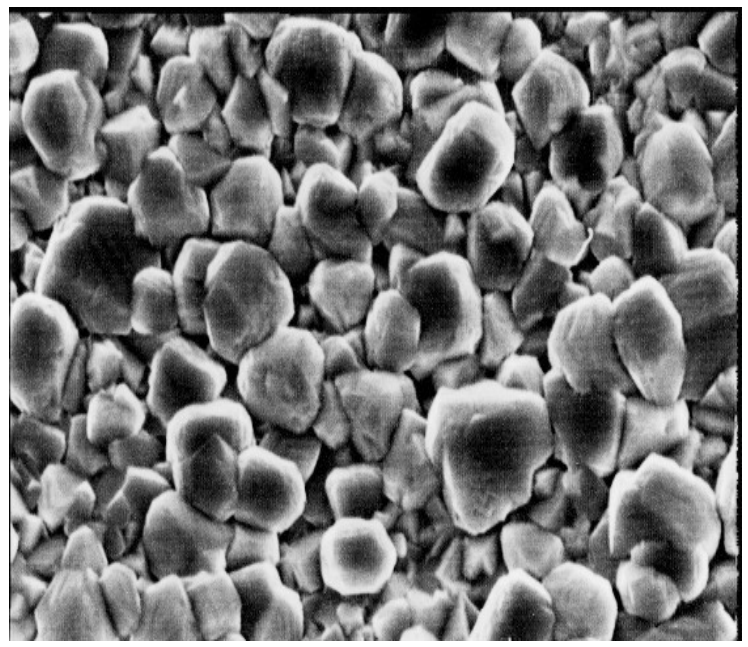

(d) corriente continua, $\mathrm{Co}_{\mathrm{d}}=0,50 \%$

$10 \mu \mathrm{m}$

Figura 3. Microestructura de los co-depósitos ZnCo. j = 30 mA.cm-2. 
La morfología de los depósitos no dependió de la densidad de corriente utilizada pero sí, del contenido de cobalto y confirma los datos experimentales obtenidos en otro trabajo [12]. Se observó también, que los recubrimientos tenían granulometría más fina si al mantener constantes las densidades de corriente de pulso y de corriente media, se aumentaban simultáneamente los valores de on-time y off-time.

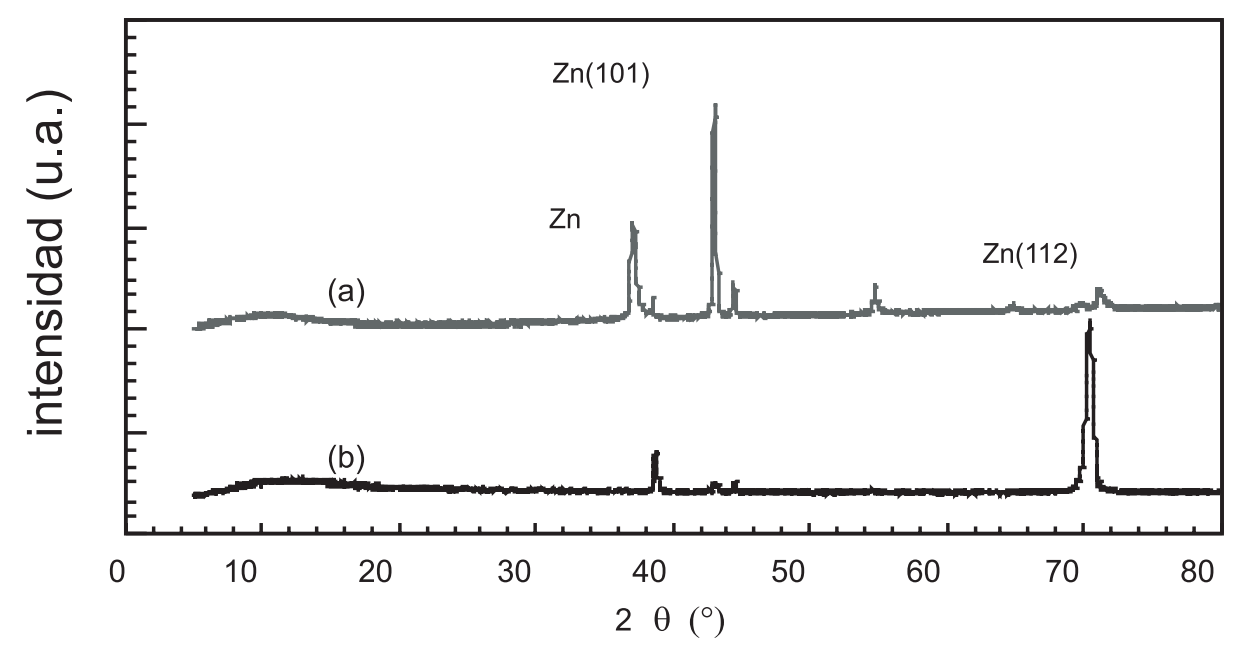

Figura 4. Difractogramas de los codepósitos de ZnCo obtenidos con: (a) corriente modulada ( $\mathrm{t}_{\text {on }}=3,2$ $\left.\mathrm{ms}, \mathrm{t}_{\text {off }}=16,8 \mathrm{~ms}, \mathrm{j}_{\mathrm{m}}=30 \mathrm{~mA} \cdot \mathrm{cm}^{-2}\right) ;(\mathbf{b})$ corriente continua $\left(\mathrm{j}=30 \mathrm{~mA} \cdot \mathrm{cm}^{-2}\right)$.

En los difractogramas de las muestras (figura 4), puede verse que la estructura cristalina es la del Zn y concuerda con las observaciones de Fratesi et al [16]. Se consideró la condición c (corriente pulsante y tenor de $\mathrm{Co}_{\mathrm{d}}=0,90 \%$ ), para comparar con la condición $\mathrm{d}$ (corriente continua y tenor de $\mathrm{Co}_{\mathrm{d}}=0,45 \%$ ).

El recubrimiento obtenido con corriente continua no era monocristalino y el pico más intenso corresponde al plano cristalino (112) del Zn (figura 4b), mientras que en la figura $4 a$ al (101). Por consiguiente, se infiere que la estructura de las muestras en examen, con tenores de Co en el depósito comprendidos entre $0,45 \%$ y $0,90 \%$, es la del cinc porque los picos revelados son atribuibles a él. Picos de cobalto no se observan debido probablemente, a la baja solubilidad del Co en Zn [17].

La tabla 1 presenta los resultados de los ensayos de microdureza realizados en los recubrimientos de $\mathrm{ZnCo}$ que se obtuvieron con las dos técnicas de electrodeposición. El análisis de los mismos permite asumir que en concordancia con lo publicado por Fratesi [16], el aumento de la microdureza está relacionado con la disminución del tamaño de los granos cristalinos, con el aumento de la concentración de Co en el depósito y con la modificación de la orientación del crecimiento cristalino del Zn (figura 4). 


\section{CONCLUSIONES}

El análisis de recubrimientos de ZnCo obtenidos mediante la aplicación de dos técnicas distintas (corriente continua o pulsante) permitió concluir que:

- Utilizada para producir un depósito con la misma concentración de Co, la concentración de aditivos que se uso en el baño, puede ser un $50 \%$ menor que la empleada con corriente continua;

- Con corriente pulsante, es posible generar un depósito con un mismo y hasta mayor contenido de Co, pero con una concentración inferior de aditivos en el baño;

- Independientemente de la técnica utilizada, los resultados relacionados con la adherencia de los recubrimientos fueron satisfactorios;

- El aumento de la microdureza está asociado con la disminución del tamaño de grano cristalino, con el aumento de la concentración de Co en el depósito y con la modificación de la orientación del crecimiento cristalino del cinc;

- La electrodeposición con corriente pulsante provoca una significativa alteración de la estructura del depósito. En este sentido, fue posible obtener depósitos con granos más finos y valores más elevados de microdureza y de cobalto.

Es importante enfatizar que al utilizar corriente pulsante para obtener electrodepósitos, los factores determinantes no son sólo la densidad de corriente media y el duty cycle, sino que también existen otros para tenerse en cuenta. En este sentido, la figura 3 muestra que con una misma densidad de corriente media y duty cycle, se logran diferentes electrodepósitos, razón por la cual, los on-time y off-time deben ser considerados como los más importantes.

\section{AGRADECIMIENTOS}

Esta investigación fue financiada por el CNPq/PROSUL (Processo 490116/2006-0), de Brasil, CAPES/MINCyT (Processo 158/09 de Brasil y BR/08/04 de Argentina), la Comisión de Investigaciones Científicas de la Provincia de Buenos Aires (CICPBA), y el Consejo Nacional de Investigaciones y Técnicas (CONICET), de Argentina.

\section{REFERENCIAS BIBLIOGRÁFICAS}

[1] Townsend, H.Y. (1991) Materials Performance, v. 30, n. 10, p. 60-65.

[2] Crotty, D. (1997) Plating Surface Finishing, v. 84, n. 4, p. 57-61.

[3] Ariga, K., Kanda, K., (1980) Tetsu to Hagane, Journal Iron Steel Inst. Japan, v. 66, p. 797-802. 
[4] Shastry, C.R., Townsend, H.Y. (1989) Corrosion, v. 45, n. 2, p. 103-119.

[5] Fratesi, R., Roventi, G., Giuliani, G., Tomachuk, C.R. (1997) Journal of Applied Electrochemistry, v. 27, p. 1088-1094.

[6] Natorski, T.J. (1992) Metal Finishing, v. 90, n. 3, p. 15-17.

[7] Porter, F.C. (1991) Zinc Handbook, Marcel Dekker Inc., New York.

[8] Freire, C.M.A., Ballester, M. (1998) Tratamento de Superfície, n 9-10, p. 30-34.

[9] Puippe, J. C., Leaman, F. (1986) Theory and Practice of Pulse Plating, American Electroplaters and Surface Finishers Society, New York.

[10] Devaraj, G.; Guruviah, S. (1990) Materials Chemistry and Physics, v. 25, n. 5, p. 439-461.

[11] Puippe, J.Cl., Ibl, N. (1980) Plating and Surface Finishing, v. 67, p. 68-72.

[12] Tomachuk, C.R.; Freire, C.M.A.; Ballester, M.; Fratesi, R., Roventi, G. (1999) Surface and Coating Technology., v. 122, n. 1, p. 6-9.

[13] Pagotto Jr., S.O.; Freire, C.M.A. y Ballester, M. (1999) Surface and Coatings Technology, v. 122, p. 10-13.

[14] Paatsch, W. (1987) Metalloberfläche, v. 41, p. 39-43.

[15] Sard, R. (1987) Plating and Surface Finishing, v. 74, n. 2, p. 30-34.

[16] Fratesi, R., Roventi, G. (1989) Materials Chemistry and Physics, v. 23, p. 529540.

[17] Hansen, M. (1958) Constitution of Binary Alloys, MacGraw Hill, p. 520. 